
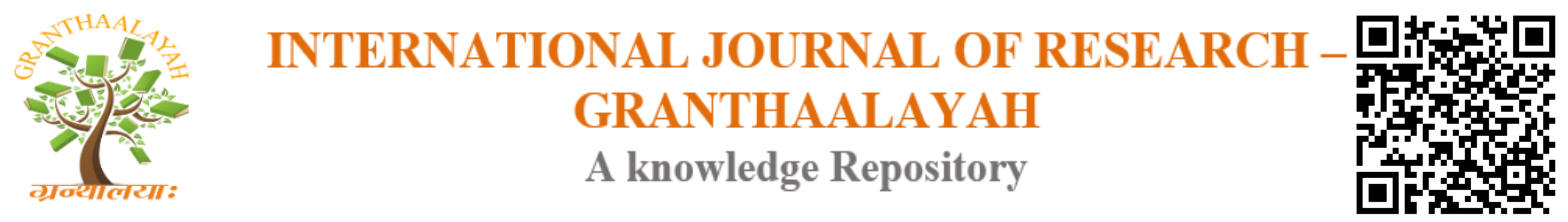

Management

\title{
PROJECT TEAM FORMATION AND DEVELOPMENT BASED ON THE IMPOVED COMPETENCE METHOD
}

\author{
G. D. Kostina ${ }^{1}$, A. V. Torgasheva ${ }^{2}$ \\ ${ }^{1,2}$ Moscow Institute of Electronic Technology - Technical University, Russia
}

\begin{abstract}
Creation of a project team is the first stage in the long sequence of all works which are related to the desired project. At this stage, a group of participants should be carefully chosen, based not only on their experience in the project area, but also on their compatibility and willingness to work together in a mutually helping way. In the paper, after a survey of related publications, project team types and characteristics are considered and arising problems are identified. These problems are related mainly to badly specified duties of team members, weak information links between them, fuzzy project goal and competition for dominance between members, It is found, that the project team formation can be viewed as a mathematically complex multi-criteria optimization task. A new method of team formation is proposed, which does not require deep mathematical background from the project manager. The method is based on the use of the competence technique. However, in contrast with the use of this technique in human resource management, the proposed method takes into account the important aspect of psychological compatibility of team members, which can appear to be the decisive factor in the project success. The method is presented as a simple algorithm which can be used by the project manager in the team formation.
\end{abstract}

Keywords: Projects; Project Team; Team Types; Team Formation; Improved Competences.

Cite This Article: G. D. Kostina, and A. V. Torgasheva. (2018). "PROJECT TEAM FORMATION AND DEVELOPMENT BASED ON THE IMPOVED COMPETENCE METHOD." International Journal of Research - Granthaalayah, 6(5), 334-339. https://doi.org/10.29121/granthaalayah.v6.i5.2018.1478.

\section{Introduction}

Development and implementation of sufficiently large projects in all areas of industry, business and technology involves a number of participants to finish the projects with available resources on time and with required quality. Participants which are involved in such work create a project team. According to the definition of J.R. Katzenbach and D.K. Smith (1993), a project team is a small group of people with complementary skills who are committed to a common work for which they are mutually accountable. In subsequent publications of M.C. Jones and A.W. Harrison (1996), C.P. Hargrave and E. Ostler (1998) and S. Gherardi, D.Nicoli and F.Odella (1998), different aspects of organization of project teams are considered in the further detail. Recent works of V.Casey (2010), B. Hnatkowska (2013) and H.Gharaibeh (2015) indicate that aspects of formation 
of project team remain a hot research topic. In particular, the survey of R.Akram, S.Ihsan and B.Hayat (2018) covers the concepts of project teams with respect to the development of software. In this area of technology, it is especially important to create such a project team which could finish the desired project in due time, with given budget and suitable quality.

During project implementation, participants are usually organized in a coordinated team and interact with each other to exchange related data and materials and to synchronize their works according to a preliminary designed and approved schedule. Therefore, logically the work of a project team is equivalent to the operation of a distributed information system. Such a system consists of a set of autonomous computing processes, that operate at different geographical sites, are involved in performing a common data processing work and coordinate their operations with the use of messages exchanged via a computer network (G. Coulouris, J. Dollimore and T. Kindberg (2001)). Clearly, project team members can be viewed as computing processes in distributed system, they are involved in a common project and interact with each other with the use of different types of communication such as telephones, electronic mail, Skype P2P system and so on. During this interaction they exchange project-related documents (data in terms of a distributed system) and agree on some form of coordination.

Traditionally, project teams are created manually by a person assigned as a project manager. Currently, especially for large project, this is more often done with the use of some computerassisted tool such as using software agents (J. Whatley (2004)) or fuzzy-genetic method (D.Strnad and N.Guid (2010)).

However, despite the considerable progress in approaches to create project teams, the further studies are necessary in this area. In current works on project team, usually relatively small teams are considered. New approaches are required to form teams for large-sale projects, where teams can be not only large, but team members can be quite different in their capabilities and psychological compatibility (M.C.Jones (2008)). The last aspect is usually not given sufficient attention in existing methods of project team formation. In this paper, a new approach is proposed and informally described, which is based on the use of competence method (H.Itami and Th.W. Roehl (1991)). Originally, this method has been oriented to planning a successful corporate strategies, with the use of so called "invisible assets" (technical know-how, the visibility of a brand-name, knowledge of a customer base) and tangible assets (people, materials, equipment and money). However, behavioral and inter-personal characteristics of team members were not given a proper attention.

In what follows, project characteristics and team types are outlined. Then the proposed method of project team formation is introduced. Finally, the related steps of the method are presented as a simple algorithm. The conclusion summarizes the proposed procedure to be used as a guide by project team developers.

\section{Project Characteristics, Team Types and Existing Problems}

Projects, their types, sizes and goals and methods of formation of project teams greatly depend on the application area and on the character of companies conducting the projects, and vary in a wide range (Jones, M.C. (2008), Fu. R., Subramanian, A., and Venkateswaran, A. (2015)). 
Correspondingly, team success in the implementation of a project depends on the team level. Dependence of team effectiveness on team level has been studied by J.R. Katzenbach and D.K. Smith (1993). In that work, the authors proposed five team levels, starting from a pseudo team up to a high-performance team. In particular, in a high-performance team, the relationships between team members and their commitment to one another's personal growth and development play the main role in the project success. On the other hand, a pseudo team is at the lowest level, where members believe that they are part of a team, but not acting as one group in a coordinated way in the project.

In the often cited work of Belibin, R.M. (2010), nine team types have been identified and described. In each of these types, the author states a positive contribution to the underlined project and an allowable weakness. In this classification, a team member is not placed in one specific category. Instead, a member can belong, for example, mainly to the category of a resource investigator and, at the same time, have a strong tendency towards the category of implementer.

The current approaches to project team formation do not pay a proper attention to a number of important problems in the work of teams. We identify the following of the related problems:

a) Badly specified roles and duties of members,

b) Weak information links between members in the team,

c) Non-adequate involvement of members in the project,

d) Lack of interaction between members during development of the project,

e) Non-uniform distribution of loads among members of the team,

f) Fuzzy project goal and the way of its achievement,

g) Struggle for dominance in the team,

h) Low cultural environment in the team.

In a particular team, only some of these problems can prevail, with less influence or even the absence of the other ones. A detailed analysis of these problems and their relevance to the intended project should be the first step in the formation of efficient and successful team. Based on the analysis of data from 135 teams, J. McDonald (2005) shows that the project cost estimates depend on two main factors - the average experience of members in the team and the experience of members in similar projects. It is interesting that, in a team with less experience of its members, project cost can be low. The reason is that such a team often does not reflect in the project some important tasks that are taken into consideration by more experienced teams and that they underestimate durations of the remaining tasks.

One of the reasons for a project to be of low quality or unsuccessful is a low psychological climate in the project team (S.T. Acuna, M. Gomez and N. Juristo (2008)). The data collected from 35 small teams indicate that high team vision preferences and high safety perceptions of team members provide significantly better project outcome. It was deduced also that tracking team climate in the team was one of indicators of the quality of product delivered by the team.

\section{Proposed Procedure of Project Team Formation}

In this paper, a new approach to project team formation is proposed and described. This approach is based on the use of the improved competence method. In its original form, this method was 
considered by H. Itami and Th. W. Roehl (1991). It is mainly applied to the area of human resource management in companies. Unfortunately, as proposed, the method cannot be used directly to project team formation.

Indeed, with human resource management, the choice of employees is usually done strictly individually for each hired worker. That is, the worker is chosen by taking into consideration mainly his or her suitability for the company, without association of the person with a specific project. Since here is only one person considered at a time, such a choice is logically similar to a single-criterion optimization problem and can be formally solved by well known optimization methods.

However, in project team formation each person to be employed is considered not only from his suitability for the company in general, but also by taken into account different criteria related to a specific project. One of the most important criteria here is the capability of the hired person to be not only a specialist in the project topic, but also to be compatible with other members of the project.

As a result, project team formation appears to be a multi-criteria optimization problem, for solution of which rather complex mathematical methods should be used. There are known attempts to solve multi-criteria optimization problems which imply rather sophisticated mathematical tools. There are more simple methods to transform the multi-criteria optimization problem into a singlecriterion task. One approach of this kind is described by G.D.Kostina, S.A.Kostina and I.O. Chudaikin (2014). However, all these methods also require a good background in mathematics for project team developers to use them practically.

We propose a rather simple method of project team formation which is more appropriate for practical use by small and medium-sized companies. In this method, project team formation is organized as a multi-stage procedure.

- At stage 1, on the base of project goals, a general model of competencies and role profile of the team is formulated.

- Stage 2 is related to the detection of additional competencies and roles with respect to the project goal.

- At stage 3, using results of the previous stage, a decision is made on the way to acquire the absent competencies for team members and extend their roles.

Finally, at stage 4, the necessary characteristics of team members, related to their psychological compatibility and ability to work in the project as one coordinated group are specified. These characteristics, together with worker's knowledge of project area, will serve as an important guide during consideration of each potential candidate for the project team.

The outlined stages of the procedure should be typically organized and supervised by the project manager assigned by the company for the corresponding project. In general, the manager should clearly understand not only the overall project goal, but also the main problems in the project and see the ways of their possible solution. However, the manager does not need to possess detailed knowledge of all project aspects since these aspects can be difficult to predict during the project team formation. 


\section{Steps of the Proposed Procedure}

In this section, the stages of the project team formation, outlined in the previous section, are extended to an informal algorithm represented in in a high-level pseudo-code, with the numbered steps and comments in round brackets where necessary. These steps are as follows.

1) Choose a group of experts. (This group can estimate in more detail some aspects of the project).

2) Develop a set of competencies necessary for the desired project. (For the development of such a set, the known contents analysis can be applied).

3) Develop behavioral factors for each competency found at Step 2. (This work should be done by the expert group chosen at Step 1).

4) For the factors, developed at Step 3, specify typical patterns of team behavior.

5) Reconsider the competencies found at Step 2.

6) Specify characteristics of each competency. (This can be done with the use of so called behavioral metric which will characterize the extent or weight of each competency from the point of view of the project). .

7) Estimate individually potential candidates for membership in the project team. (At this step, the expert group can be used. It is highly important to take into account psychological characteristics of candidates and especially their compatibility and ability to work in a mutually helping way for the project goal).

8) Prepare and evaluate a list of the candidates for the project team. (This list will be used for the final decision by the project manager, possibly together with the administration of the company).

9) Return to Step 2 for the repetition of the algorithm if the obtained list of candidates is not satisfactory. (This can be done based on the results of evaluation of obtained list at Step 8).

10) Prepare the final list of candidates. (This list can contain all related information, such as addresses of candidates for their formal invitation to participate in the project).

11) End.

The presented algorithm is informal. However, it reflects all major steps to be done in the creation of a project team. If necessary, it can be extended to include such aspects as a preliminary announcement on the project to attract attention of potential candidates. As a result of such an announcement, a complete list of willing participants can be registered. Then the complete list can be used to prepare a short list for the above described algorithm.

\section{Conclusion}

A number of current approaches to project team formation are considered here. The method proposed in this paper is based on the use of team competencies, with consideration of psychological compatibility of members with regards to the project goal. This is an important addition to the known method of competencies. The proposed method does not require deep mathematical background from project team developers. At the same time, it can be used practically for real-world applications by small and medium-sized companies. The use of the method is guided by the algorithm which shows the required steps of its application by team developer and underlines the importance of taking into account not only competencies of potential 
team members, but also their compatibility and ability to work as a group of participant supporting each other.

\section{References}

[1] Acuna, S., Gomez, M. and Juristo, N. (2008). Towards understanding the relationship between team climate and software quality: a quasi-experimental study, Empirical Software Engineering, 13(4), 401-434.

[2] Akram, R., Ihsan, S., Zafar, S. and Hayat, B. (2018). Survey Paper for Software Project Team, Staffing, Scheduling and Budgeting Problem, International Journal of Advanced Computer Science and Applications, 9(1), 479-484.

[3] Belbin, R.M. (2010). Team at work, $2^{\text {nd }}$ edition, Routledge Publ.

[4] Casey, V. (2010. Virtual software team project management, J. Braz. Comp. Soc., 16(2), 83- 96.

[5] Chen, S.-J. (2005). An Integrated Methodological Framework for Project Task Coordination and Team Organization in Concurrent Engineering, Concurrent Engineering: $R \& A, 13(3), 185-197$.

[6] Coulouris, G., Dollimore, J., Kindberg, T. (2001). Distributed Systems: Concepts and Design, Pearson Education.

[7] Fu, R., Subramanian, A. and Venkateswaran, A. (2016). Project Characteristics, Incentives, and Team Production, Management Science, 62(3), 785-801.

[8] Gharaibeh, H. (2015). A Conceptual Framework to Improve Project Team Learning

[9] in Major Projects, IJITPM, 6(2), 61-76.

[10] Gherardi, S., Nicolini, D. And Odella, F. (1998). Toward a social understanding of how people learn in organizations.Management Learning Journal, 29(3), 273-297.

[11] Hargrave, C.P. and Ostler, E. (1998). Project Team: A Collaborative Internet, Proceedings of Society for Information Technology \& Teacher Education International Conference 1998, 10631067.

[12] Hnatkowska, B. (2013). Software Engineering Team Project: Lessons Learned, e-Informatica, 7(1), $77-85$.

[13] Itami, H. and Roehl, Th.W. (1991). Mobilizing Invisible Assets, Harvard University Press.

[14] Jones, M.C. and Harrison, A.W. (1996). IS project team performance: An empirical assessment, Information \& Management, 31(2), 57-65.

[15] Jones, M.C. (2008). Large scale project team building: beyond the basics, Communications of the ACM, 51(10), 113-116.

[16] Katzenbach, J.R. and Smith, D.K. (1993). The Wisdom of Teams: Creating the High-Performance Organization, Harvard Business School, Boston.

[17] Kostina, G.D., Kostina, S.A. and Chudaikin, I.O. (2014). Reduction of a Multicriteria Optimization Problem to a Single Criterion Task, Academic Research International, 5(3), 12-17.

[18] McDonald, J. (2005). The Impact of Project Planning Team Experience on Software Project Cost Estimates, Empirical Software Engineering, 10(2), 219-234.

[19] Pons, D. and Haefele, S. (2016). Team Interactions for Successful Project Management in Small and Medium-Sized Enterprises, IJITPM, 7(2), 17-43.

[20] Silvia T. Acuna, S.T., Gomez, M. And Juristo, N. (2008). Towards understanding the relationship between team climate and software quality: a quasi-experimental study, Empirical Software Engineering, 13(4), 401-434.

[21] Strnad, D. and Guid, N. (2010). A fuzzy-genetic decision support system for projec team formation, Appl. Soft Comput., 10(4), 1178-1187.

[22] Whatley, J. (2004). Software Agents for Supporting Student Team Project Work, ICEIS, 190- 196.

*Corresponding author.

E-mail address: fmr@ miee.ru 